\title{
Cardiac magnetic resonance in myocardial disease
}

\author{
Udo Sechtem, Heiko Mahrholdt, Holger Vogelsberg
}

Heart 2007;93:1520-1527. doi: 10.1136/hrt.2005.067355

For a number of patients it is difficult to diagnose the cause of cardiac disease. In such patients cardiac magnetic resonance is useful for helping to make a differential diagnosis between ischaemic and dilated cardiomyopathy; identifying patients with myocarditis; diagnosing cardiac involvement in sarcoidosis and Chagas' disease; identifying patients with unusual forms of hypertrophic cardiomyopathy and those with continuing myocardial damage; and defining the sequelae of ablation treatment for hypertrophic obstructive cardiomyopathy.

of ischaemic scar or hibernation. A perfect demonstration of an ischaemic cause of heart failure requires demonstration of an ischaemic scar or hibernation, in addition to depicting coronary morphology by coronary angiography or computed tomography. ${ }^{1}$ Perfusion defects shown by nuclear myocardial perfusion imaging usually indicate an ischaemic scar, but such defects can also by found in patients with DCM. Segmental wall motion abnormalities are the mainstay of the echocardiographic differentiation, but they may not always distinguish between the two entities. ${ }^{2}$ Myocardial tissue differentiation by echocardiography is still in its infancy and relies on indirect signs such as differences in strain rate. ${ }^{3}$

Late gadolinium enhancement (LGE) is a new tool provided by CMR. ${ }^{4}$ LGE depicts areas of myocardial necrosis and fibrosis with high spatial resolution and accurately reflects "true" changes demonstrated by pathology both in animal models ${ }^{4}$ and human disease. ${ }^{5}$ When appropriate pulse sequences optimised to reflect differences in the spin-spin relaxation time $\mathrm{T} 2$ are used, CMR may also be able to depict tissue oedema. ${ }^{6}$ Oedema often accompanies inflammatory myocardial disease. $^{7}$

How can CMR be used for distinguishing between patients with ischaemic and non-ischaemic heart failure? Ischaemic scar is either subendocardial or transmural. ${ }^{8} \mathrm{CMR}$ is sensitive in detecting even small amounts of subendocardial infarction. ${ }^{9}$ Thus it is also sensitive in detecting ischaemic heart failure as some degree of scar will usually be present in ischaemically injured, poorly contractile ventricles. On the other hand, subendocardial enhancement should be absent in patients with normal coronary arteries and left ventricular dysfunction. Indeed, several groups have now confirmed that LGE-CMR finds scar tissue in almost all patients with a previously identified infarct related artery but only rarely in patients with non-ischaemic cardiomyopathy or healthy volunteers (table 1). ${ }^{10-12}$ Peculiar findings seen in patients without coronary artery disease are longitudinal striae of mid-wall enhancement, clearly different from the subendocardial or transmural enhancement pattern seen in patients with coronary artery disease (fig 1).

Recently, the mid-wall LGE pattern was found to be associated with active or borderline myocarditis by Dallas criteria ${ }^{13}$ in patients with a clinical presentation of chronic myocarditis and depressed left ventricular function or repetitive ventricular

Abbreviations: CMR, cardiac magnetic resonance; DCM, dilated cardiomyopathy; EMB, endomyocardial biopsy; $\mathrm{HCM}$, hypertrophic cardiomyopathy; LGE, late gadolinium enhancement; LV, left ventricle; PVB19, parvovirus B19

Accepted 21 May 2006

6 June 2006
In patients with symptoms and signs of heart failure of recent onset, echocardiography often discloses an enlarged, poorly contractile left ventricle (LV) and sometimes also signs of right report chest pain the differential diagnosis of the cause of heart failure is broad. The challenge is to distinguish between ischaemia, infection, inflammation or "idiopathic" disease. In patients without a typical history of coronary artery disease with myocardial infarction an ischaemic cause of ventricular dysfunction is still commonly ruled out by routine coronary angiography. The assumption is that the finding of stenosed or occluded coronary arteries is synonymous with the presence 
Table 1 CMR distinguishes between ischaemic and non-ischaemic cardiomyopathy

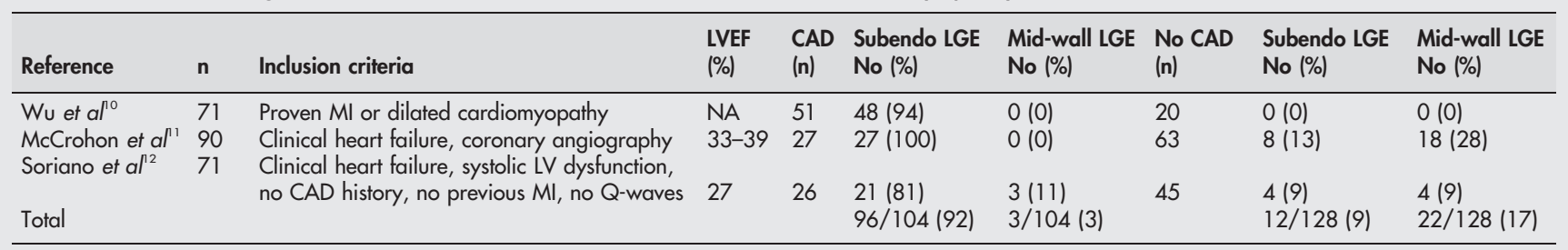

CAD, coronary artery disease; LVEF, left ventricular ejection fraction; MI, myocardial infarction; Subendo, subendothelial.

arrhythmias. ${ }^{14}$ This pattern was seen just as often in patients with active myocarditis $(43 \%)$ as in those with borderline myocarditis (44\%). Interestingly, patients with a clinical diagnosis of dilated cardiomyopathy and mid-wall LGE have a worse prognosis, as measured by unplanned admission to hospital or death, than patients with a similar degree of left ventricular dysfunction but no mid-wall LGE (Prasad S, unpublished data).

What is the histological basis of such non-endocardial myocardial enhancement in patients with DCM which does not correspond to the perfusion bed of a coronary artery? Although inflammation seems to play a part with such intramyocardial or epicardial changes, ${ }^{14}$ inflammation is unlikely to be the sole cause of LGE. As in LGE associated with infarction, an important mechanism of enhancement is the expansion of extracellular space associated with myocardial necrosis ${ }^{14}$ and later fibrosis. ${ }^{15}$ Myocyte necrosis is a feature of acute myocarditis as defined by the Dallas criteria, and myocardial fibrosis is usually found at postmortem examinations in patients with a diagnosis of DCM. Myocyte necrosis in myocarditis is usually disseminated, but larger confluent areas may exist. ${ }^{16}$ Fibrotic changes may also be diffuse or more patchy. The LGE technique is unlikely to detect diffuse necrotic or fibrotic changes as it is designed to optimise contrast between normal and necrotic myocardium in patients with focal ischaemic necrosis. ${ }^{17}$ Hence, no enhancement will be seen by LGE-CMR in patients with diffuse myocardial damage. In contrast, LGE-CMR does have sufficient spatial resolution to resolve small foci of myocardial necrosis or fibrosis in vivo. ${ }^{18}$ It is currently unknown whether the amount of fibrosis seen by CMR has prognostic value.

\section{ACUTE VIRAL MYOCARDITIS}

Clinical symptoms in patients with acute myocarditis may be absent and the only clue to the presence of the disease may be changes in the ECG. At the other end of the spectrum, patients may come to the hospital in cardiogenic shock. As myocarditis may be associated with systemic viral illness, patients may report tiredness, muscle aches, chest pain and palpitations. There are three more dramatic modes of presentation associated with myocarditis. First, myocarditis may manifest as acute onset of heart failure in a previously healthy person. Second, it may masquerade as an acute coronary syndrome. Third, arrhythmias including ventricular tachycardia may be the leading symptom, sometimes manifesting as sudden cardiac death. Establishing the diagnosis of myocarditis is difficult as there are currently no non-invasive tools to verify the diagnosis and assess the extent of myocardial involvement. The ultimate proof that the patient has myocarditis is provided by endomyocardial biopsy (EMB), which may demonstrate the typical inflammatory infiltrate in the myocardium. Using molecular hybridisation or PCR methods, it is possible to identify the causative viral agent. However, myocarditis is a patchy disease, which may explain the sampling error limiting the diagnostic value of endomyocardial biopsy. ${ }^{19}$

Friedrich et al were the first to propose CMR for non-invasive diagnosis of clinically acute myocarditis. ${ }^{20}$ They observed that the myocardium in patients with the clinical manifestations of myocarditis showed hyperenhancement relative to skeletal muscle on Tl-weighted images. However, the imaging protocol used in that study yielded a low contrast between inflamed and normal myocardium and suffered from image artefacts.

New contrast-enhanced CMR techniques such as the one employed for infarct imaging ${ }^{17}$ provide an improvement in contrast between diseased and normal myocardium of up to $500 \%$ when compared with the protocol used by Friedrich et al. When these new inversion recovery gradient echo techniques are used in patients with clinically suspected myocarditis (history of respiratory or gastrointestinal symptoms with 8 weeks of admission in combination with fatigue/malaise, chest pain, dyspnoea or tachycardia plus ECG changes such as conduction block, ST abnormalities, supraventricular tachyarrhythmia or ventricular tachycardia) LGE is found in up to $90 \%$ of the patients. ${ }^{21}$ The regions of LGE have a patchy distribution
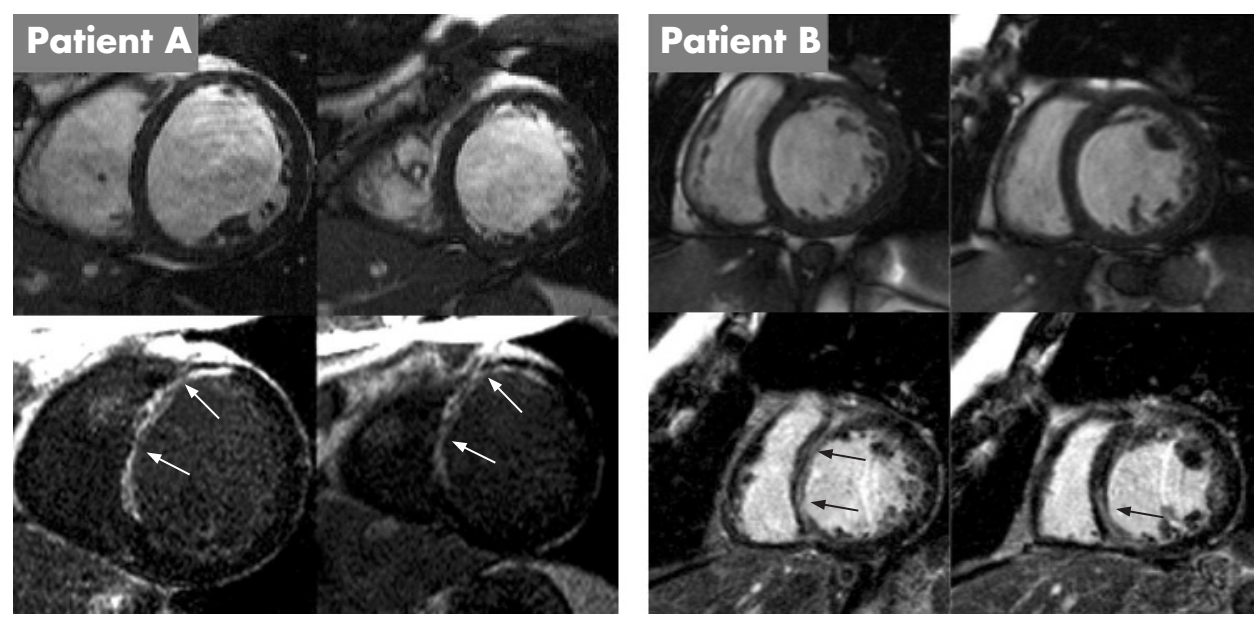

Figure 1 Short-axis images in two patients presenting with heart failure, enlarged left ventricle and systolic dysfunction. The corresponding contrast cardiac magnetic resonance images demonstrate nearly transmural late gadolinium enhancement (LGE) in the anteroseptal wall consistent with a prior myocardial infarction in the patient with ischaemic heart disease (patient $A$ ), whereas typical mid-wall stria-type LGE is present in the patient with non-ischaemic cardiomyopathy (patient B). 


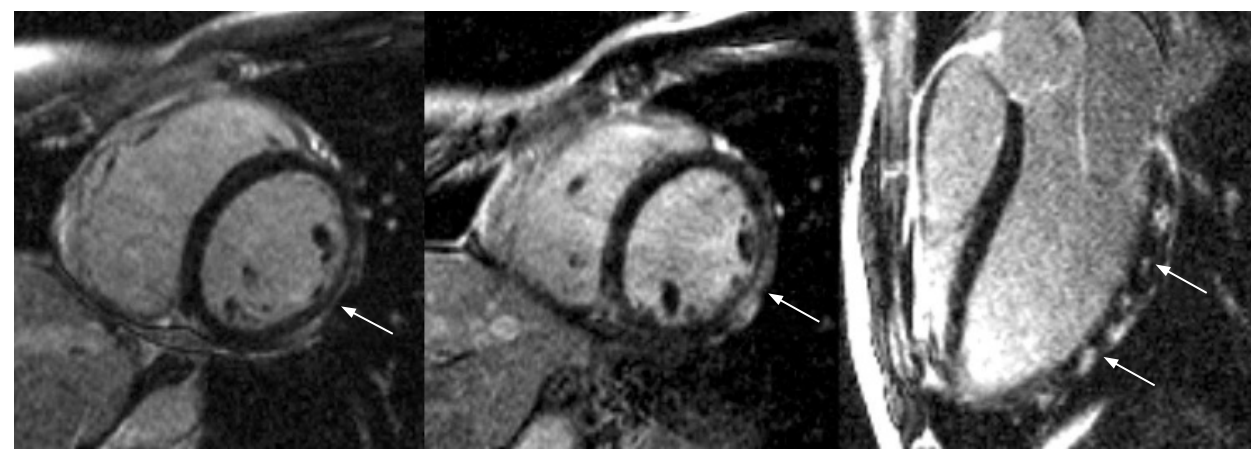

Figure 2 Left and middle panel: basal and mid-ventricular short-axis late gadolinium enhancement-cardiac magnetic resonance image in a 26-year-old man with the clinical picture of acute myocarditis demonstrating epicardial enhancement in the posterolateral aspects of the left ventricle (arrows). The three-chamber view (right panel) shows prominent bright foci of enhancement in the lateral wall (arrows). Biopsy disclosed parvovirus B19 infection. throughout the LV. They are frequently located in the lateral free wall (fig 2) and originate from the epicardial quartile of that wall. Another commonly seen pattern is the mid-wall stria pattern in the basal interventricular septum mentioned above in patients with chronic myocarditis.

Biopsy specimens obtained from the area of LGE show acute or borderline myocarditis ${ }^{13}$ in a higher percentage than reported in the literature. ${ }^{22}$ However, one has to consider that an increase in the number of activated macrophages ( $>14 /$ high power field) was an additional criterion for making the diagnosis of borderline myocarditis in the study by Mahrholdt et al. ${ }^{21}$ This represents an extension of the original Dallas criteria, ${ }^{13}$ and an increased sensitivity of EMB can hence be expected. Irrespective of this point, myocarditis is seen less consistently in patients in whom the biopsy cannot be obtained from the region of contrast enhancement (found in only one of seven patients in the study by Mahrholdt et $a^{21}$ ). Thus, CMR-guided biopsy in the right or the left ventricle may result in a higher yield of positive findings than routine right ventricular biopsy. ${ }^{23}$

As mentioned above, the inability to demonstrate diffuse myocardial changes as encountered in diffuse myocarditis with diffuse oedema formation is a disadvantage of the LGE technique. Even localised oedema without accompanying myocyte death might not result in sufficient increase in extracellular space to cause LGE. Thus, the sensitivity of LGE to detect milder forms of myocarditis may be suboptimal. Recently, it has been suggested that CMR imaging optimised at detecting inflammation or oedema may be more sensitive for identifying patients with acute myocarditis. ${ }^{24}$ Three different pulse sequences were compared in patients with cardiac symptoms such as angina, dyspnoea or palpitations accompanied by ECG changes such as ST-segment changes or conduction defects and raised serum markers. A T2-weighted triple inversion recovery pulse sequence showed a significantly higher global myocardial signal intensity in patients than in volunteers, although there was overlap. A cut-off value of 1.9 had a sensitivity of $84 \%$ and a specificity of $74 \%$ to identify the disease. A Tl-weighted spin echo before and shortly after contrast injection (as described by Friedrich et $a^{20}$ ) yielded a significantly higher global myocardial relative enhancement in patients than in volunteers. A cut-off value of 4.0 had a sensitivity of $80 \%$ and a specificity of $73 \%$ to identify myocarditis. The sensitivity of a inversion recovery gradient echo pulse sequence (LGE sequence) started 10 minutes after contrast injection was lower at only $44 \%$ (fig 3), but the specificity was high (100\%). The best diagnostic performance was obtained when any two of the criteria obtained by the three techniques were positive in a given patient. ${ }^{24}$ One needs to

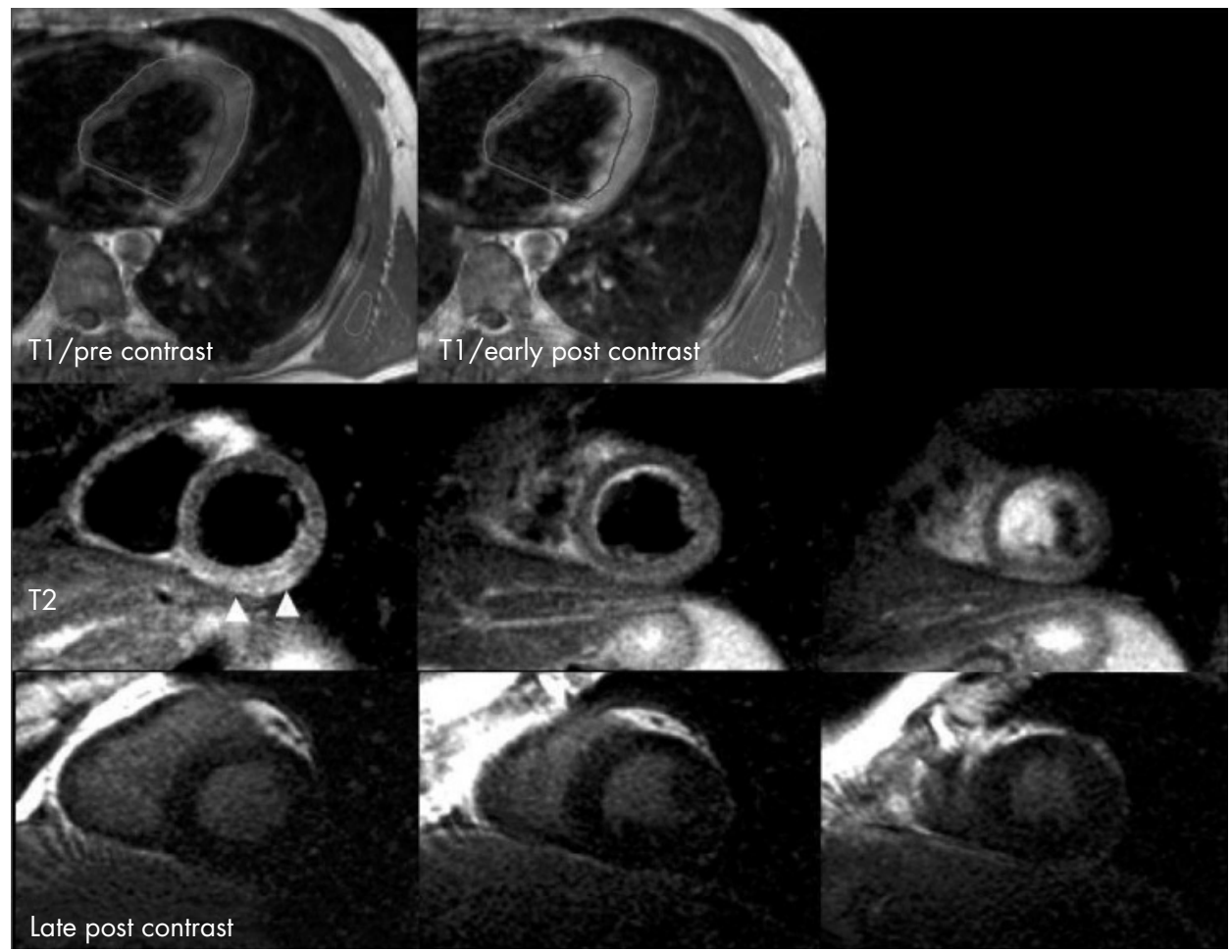

Figure 3 Cardiovascular magnetic resonance findings in a patient with acute chest pain and ST elevations in II, III, aVF as well as negative T-waves in II, III, aVF, V5V6. The patient also had a mild increase of cardiac markers. (Top) Pre- and postcontrast axial $\mathrm{Tl}$-weighted spin-echo images of the same slice. Global relative enhancement was increased (4.1). (Middle) T2-weighted images in three short-axis slices. Note the posterolateral focal high T2 signal (arrowheads) in the basal slice with apparent focal increase in myocardial thickness. (Bottom) Corresponding late enhancement images: no evidence of late gadolinium enhancement. Reprinted with permission from Abdel-Aty H, Boyé P, Zagrosek A, et al. The sensitivity and specificity of contrastenhanced and T2-weighted cardiovascular magnetic resonance to detect acute myocarditis. J Am Coll Cardiol 2005;45:1815-22. ${ }^{24}$ 


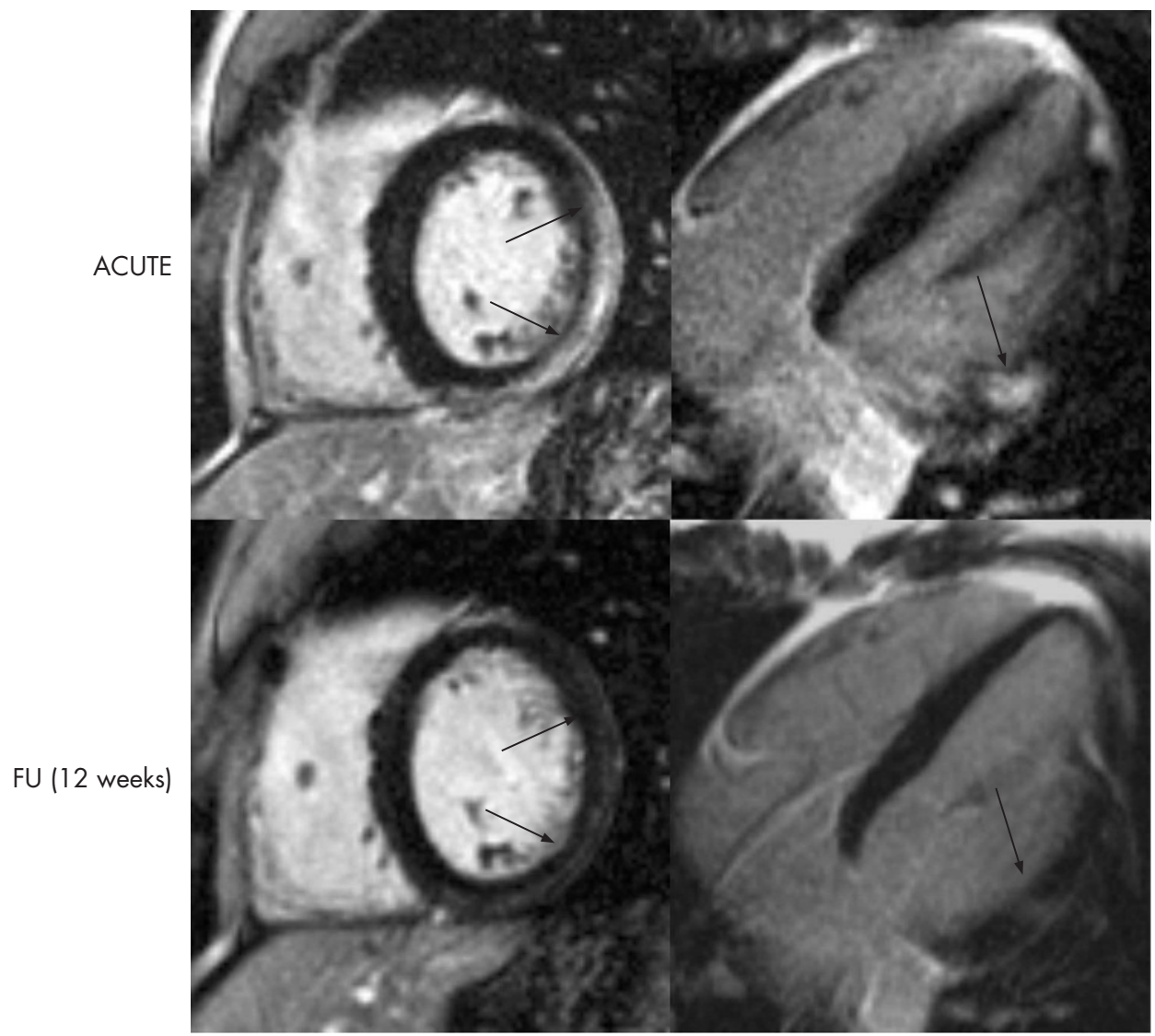

Figure 4 Serial late gadolinium enhancement (LGE) images in another patient with acute parvovirus B19 myocarditis. Upper panel: short-axis (left) and four-chamber view (right) images show prominent enhancement of the lateral wall extending almost throughout the entire wall but originating from the epicardium (arrows). Lower panel: at follow-up LGEcardiac magnetic resonance 4 months later, the inflammatory lesions have faded and become smaller. Reprinted with permission from Mahrholdt H, Goedecke C, Wagner A et al. Cardiovascular magnetic resonance assessment of human myocarditis: a comparison to histology and molecular pathology. Circulation 2004; 109:1250-8. ${ }^{21}$

remember, however, that in this series the preferred method for identifying myocarditis was the clinical presentation of the patient, and EMB was not performed.

CMR is also helpful for differentiating between acute injury due to inflammation and ischaemic injury. ${ }^{25}$ Whereas patients with acute myocardial infarction show segmental early subendocardial defects on perfusion MRI, with corresponding segmental subendocardial or transmural LGE in a vascular distribution, patients with myocarditis have no early defect and focal or diffuse non-segmental non-subendocardial LGE. Moreover, CMR gives first insights into the clinical course of patients with myocarditis. It appears that $\mathrm{Tl}$ contrast enhancement decreases to almost normal values within 4 weeks after the initial clinical presentation in most patients. ${ }^{26}$ The area of
LGE also decreases in many patients and enhancement may disappear completely (fig 4). However, the decrease in enhancement is only moderately correlated with the improvement in ejection fraction. ${ }^{21}$

In Germany, PVB19 is currently the most commonly encountered causative agent, followed by adenovirus and human herpes virus type $6 .^{21}{ }^{27}$ Parvovirus associated myocarditis often shows LGE of the epicardial portions of the free lateral wall of the LV. ${ }^{28}$ Interestingly, epicardial lesions are also the predominant findings in canine parvovirus myocarditis. ${ }^{29}$ This finding may, however, not be parvovirus specific as in the United States gross lesions have also been found in epicardial portions of the posterolateral free wall in patients dying suddenly with acute myocarditis, ${ }^{30}$ although parvovirus



Figure 5 Spin-echo (upper panel) and late gadolinium enhancement-cardiac magnetic resonance (LGE-CMR; lower panel) images in a patient with biopsy proven with pulmonary sarcoidosis. A diffuse signal is seen in both lungs on spin-echo images. LGE short-axis images (three images from the left in the lower panel from base to apex) demonstrate inferior and lateral enhancement not dissimilar to that seen in myocarditis. With steroid treatment, symptoms improved and myocardial enhancement became fainter. 


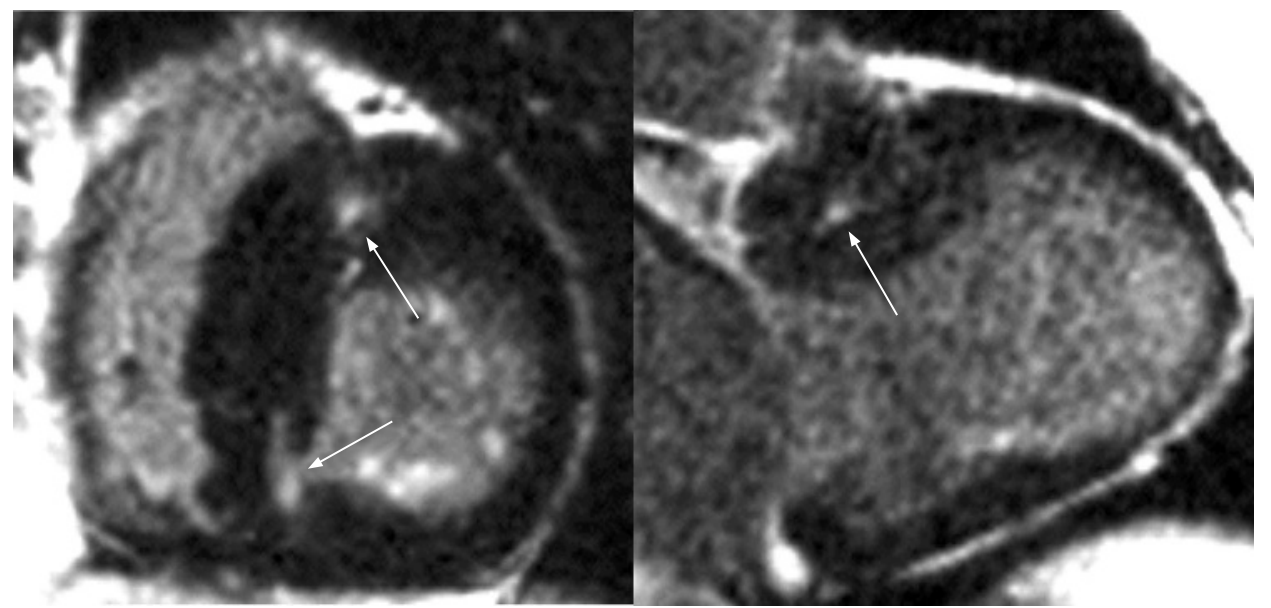

Figure 6 Patient with asymmetric hypertrophy and small scars at junctions between the left and right ventricles (arrows) shown by late gadolinium enhancementcardiac magnetic resonance. Left: short-axis image. Right: two-chamber view parallel to the interventricular septum.

infection is not common in the United States. ${ }^{31}$ In contrast, the peculiar pattern of a sandwiched stripe of late enhancement within the interventricular septum is more frequently found in herpes virus or mixed infections caused by herpes virus and parvovirus. ${ }^{28}$ Intraseptal lesions are also seen at necropsy in patients dying of active myocarditis. ${ }^{19}$

\section{OTHER FORMS OF INFLAMMATORY CARDIOMYOPATHY}

In patients with clinical signs of cardiac sarcoidosis (arrhythmias, conduction disturbances, heart block, pericardial effusion or sudden death), cine CMR usually demonstrates regional wall motion abnormalities and may also show wall thickening of the affected region. ${ }^{32}$ T2-weighted imaging may demonstrate diffuse regional signal increase. LGE often shows focal areas of inflammation or fibrosis, or both, ${ }^{33}$ (fig 5) and contrast enhancement may show patterns of epicardial enhancement similar to that seen in myocarditis. As in patients with myocarditis, findings of T2 and LGE images are not necessarily concordant. Although CMR findings may be non-specific, the high sensitivity makes CMR an attractive first-line non-invasive method for early diagnosis and follow-up of cardiac sarcoidosis.

Chagas' disease is an inflammatory form of myocardial disease caused by the protozoan Trypanosoma cruzi. Although the inflammatory myocardial process is crucial for parasite control, inflammation may become progressive, resulting in chronic disease that is characterised by myocarditis associated with prominent fibrosis and cardiac dysfunction. CMR demonstrates myocardial LGE in two-thirds of patients diagnosed with Chagas' disease, ${ }^{34}$ and the incidence increases with increasing severity of cardiac dysfunction and is most pronounced in patients with severe ventricular arrhythmias. LGE patterns may be similar to viral myocarditis, affecting the epicardial portion of the LV free wall. ${ }^{34}$ Thus, CMR enables the quantification of myocardial inflammation and fibrosis in Chagas' disease, adding important information on disease severity, and may become useful for early identification of cardiac involvement in the subclinical phases of the disease process.

\section{HYPERTROPHIC CARDIOMYOPATHY}

CMR has also become a useful technique in the management of patients with hypertrophic cardiomyopathy (HCM). Differentiating between HCM and other forms of hypertrophy has remained a substantial challenge for imaging techniques. Although most patients with HCM have a typical asymmetric pattern of hypertrophy affecting the interventricular septum more than the posterolateral wall, concentric, apical and other atypical distributions of hypertrophy do exist. These atypical forms of hypertrophy, in particular, may be difficult to recognise by echocardiography. CMR can identify such regions of LV hypertrophy and thus represents a powerful supplemental imaging test with distinct diagnostic advantages for selected patients with HCM. ${ }^{35} 36$

CMR can also be used to assess myocardial tissue characteristics in vivo by applying the late enhancement technique to patients with HCM. Myocardial scarring is a common finding in patients with HCM and may be prognostically important. Scarring occurs mostly in hypertrophied regions and is usually patchy with multiple foci, predominantly affecting the midventricular wall. ${ }^{37}$ However, the location of scarring does not correspond to the perfusion territories of the epicardial coronary arteries. It is currently not clear whether increased numbers of structurally abnormal intramural coronary arteries within areas of scarred myocardium have a causal role in producing myocardial ischaemia leading to scarring. In patients with small areas of scarring, the junctions of the interventricular septum and the right ventricular walls are commonly affected (fig 6). In patients with disease caused by mutations in the troponin I gene, focal fibrosis is usually not detected by LGE-CMR before left ventricular hypertrophy and ECG abnormalities are present. ${ }^{38}$ However, once hypertrophy is present, LGE is common and the extent correlates with adverse clinical measures. This suggests that focal fibrosis is closely linked to disease development. The extent of scarring measured by CMR correlates with conventional risk markers, but it is currently not clear whether the extent of scarring in the CMR will be more predictive of serious arrhythmic events than the current risk stratification based on risk factors.

Patients with severe forms of hypertrophic cardiomyopathy may develop progressive left ventricular impairment associated with progressive interstitial fibrosis, myocardial disarray, small vessel disease, and microscopic scarring leading to wall thinning. CMR demonstrates a greater extent of hyperenhancement in patients with clinically progressive disease and this is associated with thinning of previously grossly hypertrophied myocardium. ${ }^{39}$

Similar to (colour) Doppler echocardiography, CMR can depict and quantify the turbulent jet in the left ventricular outflow tract in patients with the obstructive form of HCM. A unique advantage of CMR is the opportunity for monitoring and quantifying the changes induced by septal ablation of the obstructive lesion (fig 7). The remodelling of the left ventricular outflow tract can be serially monitored during the healing process. ${ }^{40}$

Thus, CMR provides comprehensive information on anatomy, function and tissue composition in patients with HCM. 

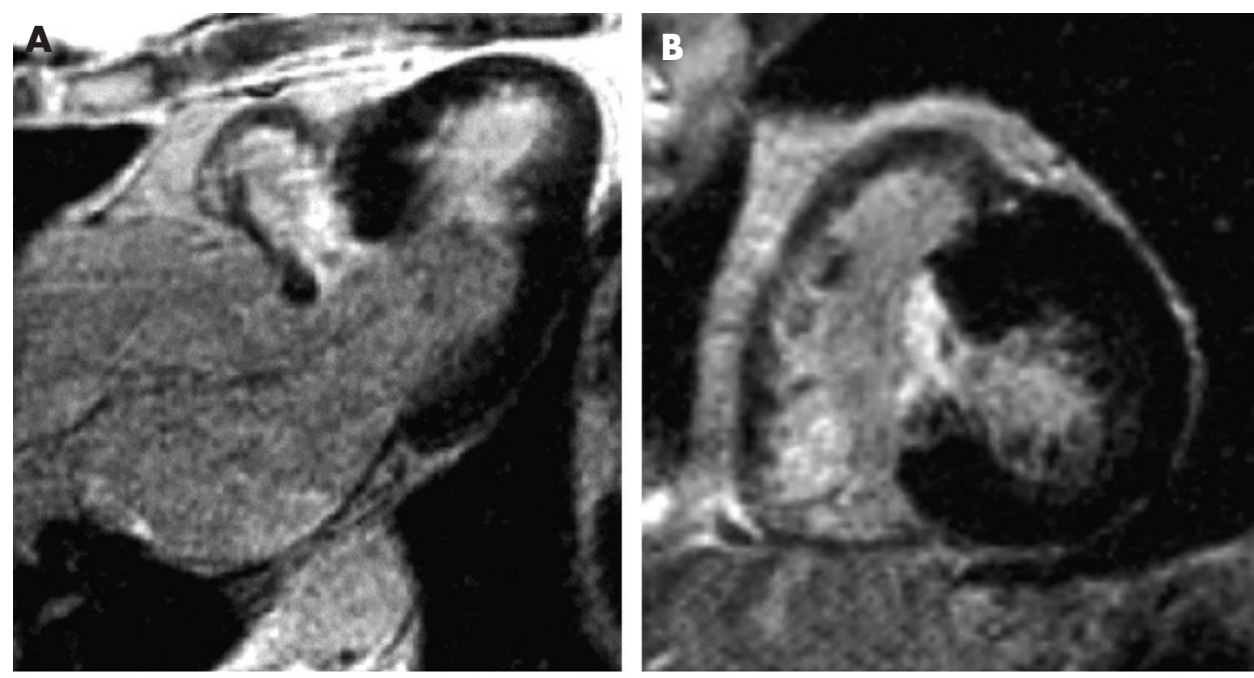

Figure 7 Contrast-enhanced images 20 minutes after intravascular administration of gadolinium-DTPA in two patients with hypertrophic obstructive cardiomyopathy 1 month after percutaneous transluminal septal myocardial ablation. (A, B) Threechamber view and short-axis view in a patient with transmural septal infarction. (C, D) Comparable views in a patient with myocardial infarction located exclusively on the right ventricular side of the interventricular septum. Reprinted with permission from van Dockum WG, ten Cate FJ, ten Berg JM, et al. Myocardial infarction after percutaneous transluminal septal myocardial ablation in hypertrophic obstructive cardiomyopathy: evaluation by contrast-enhanced magnetic resonance imaging. J Am Coll Cardiol 2004;43:27-
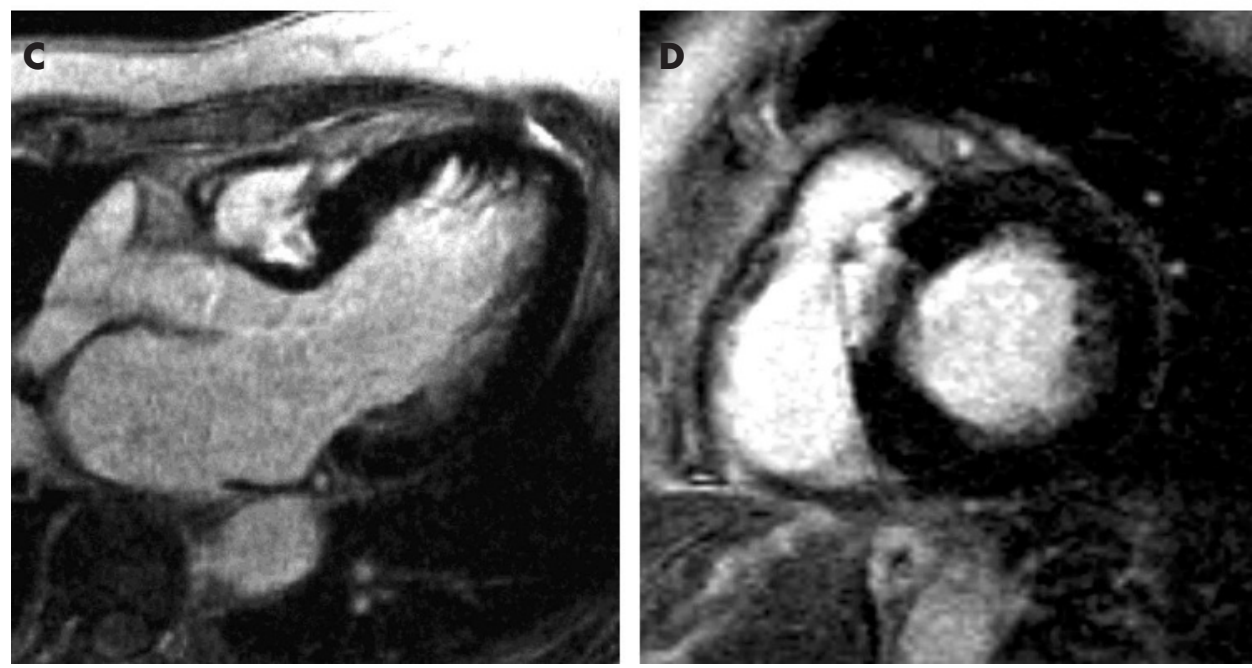
$34 .{ }^{40}$

Although the value of CMR as compared with echocardiography is not clearly defined yet, CMR will be useful in patients for whom complete information cannot be obtained by echocardiography.

\section{OTHER CARDIOMYOPATHIES}

\section{Stress-induced cardiomyopathy}

Left apical ballooning (takotsubo cardiomyopathy) is a clinical entity characterised by acute but rapidly reversible left ventricular systolic dysfunction in the absence of atherosclerotic coronary artery disease, often triggered by profound psychological stress. Catecholamine production and myocardial stunning are thought to be involved pathophysiologically and the distal portion of the LV is most commonly affected. CMR contributes to an understanding of this new entity by demonstrating the absence of irreversible injury ${ }^{41}$ (LGE) but oedema formation on T2-weighted images. CMR also aids in clearly identifying segmental wall-motion abnormalities

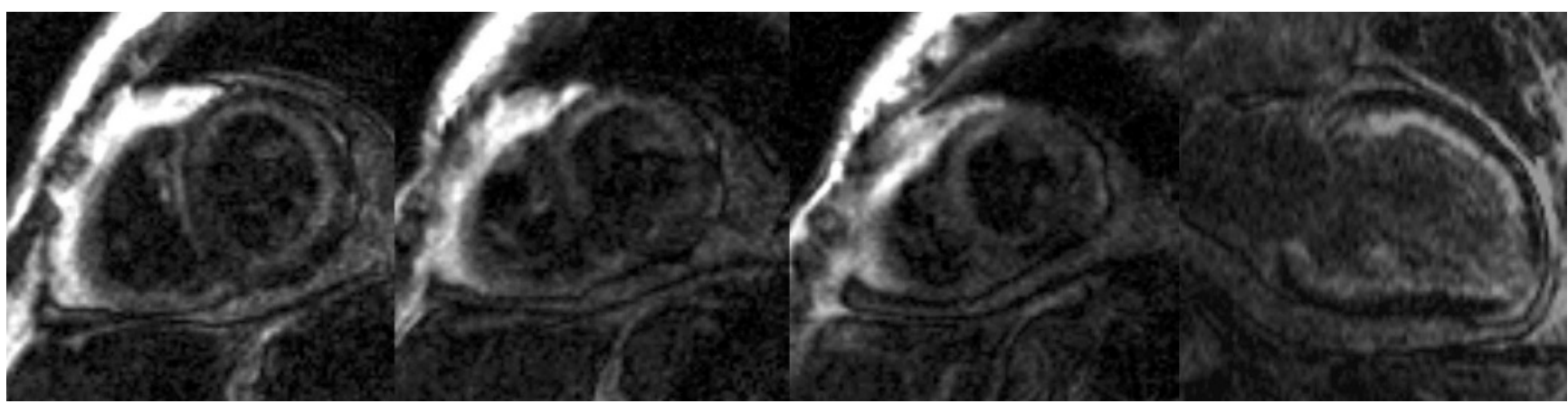

Figure 8 Subendocardial enhancement on late gadolinium enhancement short-axis (left from base to apex) and two-chamber view (parallel to interventricular septum) images in a patient with immunoglobulin light chain (AL) cardiac amyloidosis due to multiple myeloma. Note that image acquisition needs to be started earlier than the usual 10 minutes after contrast injection as the contrast agents quickly diffuse into the amyloid-filled enlarged interstitial space, and differential enhancement may be absent at later phases of the diffusion process. In patients with possible amyloid disease (eg, in all patients with unexplained left ventricular hypertrophy) and abnormal findings on TI scout images, an early series of contrast images should be acquired. 
encompassing the left ventricular myocardium in multiple coronary arterial vascular territories. ${ }^{42}$ In patients with severe haemodynamic impairment, CMR may also demonstrate involvement of the right ventricle.

\section{Infiltrative cardiomyopathies}

Cardiac involvement is common in systemic amyloidosis. It is a major determinant of treatment options and prognosis and is a frequent cause of death. Myocardial disease is often the only manifestation of transthyretin amyloidosis. Storage of proteins in the insoluble fibrillar amyloid conformation occurs principally in the myocardial interstitium, with a preference for the subendocardial space. Clinically, patients present with signs of diastolic heart failure progressing to restrictive cardiomyopathy. Relaxation time Tl measured from Tl maps is significantly lower in the subendocardial space of patients with amyloidosis than in hypertensive controls. Moreover, within patients, subendocardial $\mathrm{Tl}$ is lower than $\mathrm{Tl}$ in the subepicardium for the first 8 minutes after the administration of a gadoliniumbased contrast agent. ${ }^{43}$ This difference is no longer apparent at later time intervals. Global subendocardial LGE is seen in patients with more severe forms of the disease characterised by a greater LV mass (fig 8). Image acquisition needs to start earlier than the usual 10 minutes after contrast injection and optimal results are achieved when imaging is started after 5 minutes. The combination of this typical LGE pattern with an optimised Tl threshold between myocardium and blood has an accuracy of $97 \%$ for making the correct diagnosis. ${ }^{43}$ However, as amyloid is not always evenly distributed, a more patchy pattern of LGE may also be seen in some patients. The main mechanism of LGE in patients with amyloidosis differs from that seen in patients with chronic myocardial infarction. Whereas myocyte cell death and replacement fibrosis are responsible for the increased partition coefficient of the contrast agent in the latter, expansion of the interstitium by massive amyloid deposits without myocyte death explains the less intense LGE in amyloid heart disease.

\section{Fabry's disease}

Storage diseases are important differential diagnoses in patients with left ventricular hypertrophy. In Fabry's disease, accumulation of an abnormal glycoprotein occurs in the myocyte due to $\alpha$-galactosidase deficiency. CMR may detect regional or global myocardial thickening ${ }^{44}$ and regional LGE is seen at later and more severe stages of the disease. ${ }^{45}$ The posterolateral wall is predominantly affected but the reason for this is unknown.

\section{Haemochromatosis}

Death due to iron overload cardiomyopathy is common in countries with a large population of patients with $\beta$-thalassaemia major. Cardiac failure can be avoided if intensive chelation therapy is instituted at an early stage of myocardial iron overload. Serum iron and hepatic iron are poor substitutes for direct measurements of myocardial iron concentration. Noninvasive determination of the cardiac iron load is possible by measuring myocardial relaxation time $\mathrm{T} 2{ }^{*} .{ }^{46}$ Myocardial $\mathrm{T} 2{ }^{*}$ correlates well with cardiac iron concentration measured from biopsy specimens ${ }^{47}$ and $\mathrm{T}^{*}$ values $<20 \mathrm{~ms}$ are associated with heart failure in patients with $\beta$-thalassaemia major. T2* CMR measurements were the basis for a recent randomised trial suggesting that a new chelating agent (deferiprone) can unload myocardial iron faster than the standard chelator deferoxamine. ${ }^{48}$ Thus, regular determination of myocardial $\mathrm{T} 2{ }^{*}$ should be able to decrease the incidence of heart failure in patients with thalassaemia because aggressive chelation therapy can be started earlier.
Practical implications for CMR in myocardial disease

Patients with myocardial disease as the basis of their clinical presentation and echocardiographic findings should undergo CMR if further aetiological differentiation is felt to be of clinical value. As such patients may present with otherwise unexplained signs and objective findings of myocardial disease in the presence of a "normal" echocardiogram, CMR should also be considered in such cases. CMR may provide new anatomical information in patients with poor echo windows, but the main contribution of CMR is the LGE examination. Contrast enhancement is non-specific, but the diagnosis of cardiomyopathy can be made. The definitive diagnosis often relies on the findings of endomyocardial biopsy.

The main contributions of CMR are:

- helping to make differential diagnosis between ischaemic and dilated cardiomyopathy;

- identifying patients with myocarditis;

- diagnosing cardiac involvement in sarcoidosis and Chagas' disease;

- identifying patients with unusual forms of hypertrophic cardiomyopathy and those with continuing myocardial damage;

- defining the sequelae of ablation treatment for hypertrophic obstructive cardiomyopathy;

- helping with the differential diagnosis in patients with left ventricular hypertrophy by providing clues to the presence of infiltrative cardiomyopathies.

\section{Authors' affiliations \\ U Sechtem, H Mahrholdt, H Vogelsberg, Division of Cardiology and Pulmology, Robert-Bosch-Krankenhaus, Auerbachstrasse, Stuttgart, Germany \\ Competing interests: None declared.}

\section{REFERENCES}

1 Le T, Ko JY, Kim HT, et al. Comparison of echocardiography and electron beam tomography in differentiating the etiology of heart failure. Clin Cardiol 2000;23:417-20.

2 Duncan AM, Francis DP, Gibson DG, et al. Differentiation of ischaemic from nonischaemic cardiomyopathy during dobutamine stress by left ventricular longaxis function: additional effect of left bundle-branch block. Circulation 2003;108:1214-20.

3 Kato TS, Noda A, Izawa H, et al. Discrimination of nonobstructive hypertrophic cardiomyopathy from hypertensive left ventricular hypertrophy on the basis of strain rate imaging by tissue Doppler ultrasonography. Circulation 2004; 1 10:3808-14

4 Kim RJ, Fieno DS, Parrish TB, et al. Relationship of MRI delayed contrast enhancement to irreversible injury, infarct age, and contractile function. Circulation 1999; 100:1992-2002.

5 Moon JC, Reed E, Sheppard MN, et al. The histologic basis of late gadolinium enhancement cardiovascular magnetic resonance in hypertrophic cardiomyopathy. J Am Coll Cardiol 2004;43:2260-4.

6 Boxt LM, Hsu D, Katz J, et al. Estimation of myocardial water content using transverse relaxation time from dual spin-echo magnetic resonance imaging. Magn Reson Imaging 1993;1 1:375-83.

7 Hiramitsu S, Morimoto S, Kato S, et al. Transient ventricular wall thickening in acute myocarditis: a serial echocardiographic and histopathologic study. Jpn Circ J 2001;65:863-6.

8 Reimer KA, Lowe JE, Rasmussen MM, et al. The wavefront phenomenon of ischemic cell death. 1. Myocardial infarct size vs duration of coronary occlusion in dogs. Circulation 1977;56:786-94.

9 Wagner A, Mahrholdt H, Holly TA, et al. Contrast-enhanced MRI and routine single photon emission computed tomography (SPECT) perfusion imaging for detection of subendocardial myocardial infarcts: an imaging study. Lancet 2003;361:374-9.

10 Wu E, Judd RM, Vargas JD, et al. Visualisation of presence, location, and transmural extent of healed $Q$-wave and non-Q-wave myocardial infarction. Lancet 2001;357:21-8.

11 McCrohon JA, Moon JC, Prasad SK, et al. Differentiation of heart failure related to dilated cardiomyopathy and coronary artery disease using gadoliniumenhanced cardiovascular magnetic resonance. Circulation 2003;108:54-9. 
12 Soriano CJ, Ridocci F, Estornell J, et al. Noninvasive diagnosis of coronary artery disease in patients with heart failure and systolic dysfunction of uncertain etiology, using late gadolinium-enhanced cardiovascular magnetic resonance. J Am Coll Cardiol 2005;45:743-8.

13 Aretz HT. Diagnosis of myocarditis by endomyocardial biopsy. Med Clin North Am 1986:70:1215-26.

14 De Cobelli F, Pieroni M, Esposito A, et al. Delayed gadolinium-enhanced cardiac magnetic resonance in patients with chronic myocarditis presenting with heart failure or recurrent arrhythmias. J Am Coll Cardiol 2006;47:1649-54.

15 Mahrholdt H, Wagner A, Judd RM, et al. Delayed enhancement cardiovascular magnetic resonance assessment of non-ischaemic cardiomyopathies. Eur Heart $J$ 2005;26:1461-74.

16 Davies MJ. The cardiomyopathies: an overview. Heart 2000;83:469-74.

17 Simonetti OP, Kim RJ, Fieno DS, et al. An improved MR imaging technique for the visualization of myocardial infarction. Radiology 2001;218:215-23.

18 Ricciardi MJ, Wu E, Davidson CJ, et al. Visualization of discrete microinfarction after percutaneous coronary intervention associated with mild creatine kinaseMB elevation. Circulation 2001;103:2780-3.

19 Hauck AJ, Kearney DL, Edwards WD. Evaluation of postmortem endomyocardial biopsy specimens from 38 patients with lymphocytic myocarditis: implications for role of sampling error. Mayo Clin Proc 1989;64:1235-45.

20 Friedrich MG, Strohm O, Schulz-Menger J, et al. Contrast media-enhanced magnetic resonance imaging visualizes myocardial changes in the course of viral myocarditis. Circulation 1998:97:1802-9.

21 Mahrholdt H, Goedecke C, Wagner A, et al. Cardiovascular magnetic resonance assessment of human myocarditis: a comparison to histology and molecular pathology. Circulation 2004;109:1250-8.

22 Mason JW, O'Connell JB, Herskowitz A, et al. A clinical trial of immunosuppressive therapy for myocarditis. N Engl J Med 1995;333:269-75.

23 Mangin M, Mahrholdt H, Hager S, et al. Cardiovascular magnetic resonance assessment of human myocarditis using a standardized combined right and left ventricular endomyocardial biopsy protocol [abstract]. Circulation 2005;109(Suppl 2):145.

24 Abdel-Aty H, Boyé $\mathrm{P}$, Zagrosek $\mathrm{A}$, et al. The sensitivity and specificity of contrastenhanced and T2-weighted cardiovascular magnetic resonance to detect acute myocarditis. J Am Coll Cardiol 2005;45:1815-22.

25 Laissy JP, Hyafil F, Feldman $\amalg$, et al. Differentiating acute myocardial infarction from myocarditis: diagnostic value of early- and delayed-perfusion cardiac MR imaging. Radiology 2005;237:75-82.

26 Wagner A, Schulz-Menger J, Dietz R, et al. Long-term follow-up of patients with acute myocarditis by magnetic resonance imaging. MAGMA 2003:16:17-20.

$27 \mathrm{Kühl} \mathrm{U,} \mathrm{Pauschinger} \mathrm{M,} \mathrm{Seeberg} \mathrm{B,} \mathrm{et} \mathrm{al.} \mathrm{Viral} \mathrm{persistence} \mathrm{in} \mathrm{the} \mathrm{myocardium} \mathrm{is}$ associated with progressive cardiac dysfunction. Circulation 2005;112:1965-70.

28 Mahrholdt H, Wagner A, Deluigi C, et al. Presentation, patterns of myocardial damage and clinical course of viral myocarditis. Circulation 2006;1 14:1581-90.

29 Lenghaus C, Studdert MJ. Acute and chronic viral myocarditis. Acute diffuse nonsuppurative myocarditis and residual myocardial scarring following infection with canine parvovirus. Am J Pathol 1984;115:316-9.

30 Shirani J, Freant $\amalg$, Roberts WC. Gross and semiquantitative histologic findings in mononuclear cell myocarditis causing sudden death, and implications for endomyocardial biopsy. Am J Cardiol 1993;72:952-7.
31 Bowles NE, Ni J, Kearney DL, et al. Detection of viruses in myocardial tissues by polymerase chain reaction. evidence of adenovirus as a common cause of myocarditis in children and adults. J Am Coll Cardiol 2003:42:466-72.

32 Vignaux 0 . Cardiac sarcoidosis: spectrum of MRI features. AJR Am J Roentgenol 2005; 184:249-54.

33 Smedema JP, Snoep G, van Kroonenburgh MP, et al. Evaluation of the accuracy of gadolinium-enhanced cardiovascular magnetic resonance in the diagnosis of cardiac sarcoidosis. J Am Coll Cardiol 2005;45:1683-90.

34 Rochitte CE, Oliveira PF, Andrade JM, et al. Myocardial delayed enhancement by magnetic resonance imaging in patients with Chagas' disease: a marker of disease severity. J Am Coll Cardiol 2005;46:1553-8.

35 Rickers C, Wilke NM, Jerosch-Herold M, et al. Utility of cardiac magnetic resonance imaging in the diagnosis of hypertrophic cardiomyopathy. Circulation 2005;112:855-61

36 Moon JC, Fisher NG, McKenna WJ, et al. Detection of apical hypertrophic cardiomyopathy by cardiovascular magnetic resonance in patients with nondiagnostic echocardiography. Heart 2004;90:645-9.

37 Choudhury L, Mahrholdt H, Wagner A, et al. Myocardial scarring in asymptomatic or mildly symptomatic patients with hypertrophic cardiomyopathy J Am Coll Cardiol 2002:40:2156-64.

38 Moon JC, Mogensen J, Elliott PM, et al. Myocardial late gadolinium enhancement cardiovascular magnetic resonance in hypertrophic cardiomyopathy caused by mutations in troponin I. Heart 2005;91:1036-40.

39 Moon JC, McKenna WJ, McCrohon JA, et al. Toward clinical risk assessment in hypertrophic cardiomyopathy with gadolinium cardiovascular magnetic resonance. J Am Coll Cardiol 2003:41:1561-7.

40 van Dockum WG, ten Cate FJ, ten Berg JM, et al. Myocardial infarction after percutaneous transluminal septal myocardial ablation in hypertrophic obstructive cardiomyopathy: evaluation by contrast-enhanced magnetic resonance imaging J Am Coll Cardiol 2004:43:27-34

41 Haghi D, Papavassiliu T, Fluchter S, et al. Variant form of the acute apical ballooning syndrome (takotsubo cardiomyopathy): observations on a novel entity. Heart 2006;92:392-4.

42 Sharkey SW, Lesser JR, Zenovich AG, et al. Acute and reversible cardiomyopathy provoked by stress in women from the United States. Circulation 2005; 111:472-9.

43 Maceira AM, Joshi J, Prasad SK, et al. Cardiovascular magnetic resonance in cardiac amyloidosis. Circulation 2005:111:186-93.

44 Weidemann F, Breunig F, Beer M, et al. Improvement of cardiac function during enzyme replacement therapy in patients with Fabry disease: a prospective strain rate imaging study. Circulation 2003;108:1299-301.

45 Weidemann $F$, Breunig $F$, Beer $M$, et al. The variation of morphological and functional cardiac manifestation in Fabry disease: potential implications for the time course of the disease. Eur Heart J 2005;26:1221-7.

46 Anderson L, Holden S, Davies B, et al. Cardiovascular T2-star (T2*) magnetic resonance for the early diagnosis of myocardial iron overload. Eur Heart $J$ $2001 ; 22: 2171-9$

47 Mavrogeni SI, Markussis V, Kaklamanis L, et al. A comparison of magnetic resonance imaging and cardiac biopsy in the evaluation of heart iron overload in patients with beta-thalassemia major. Eur J Haematol 2005;75:241-7.

48 Neufeld EJ. Oral chelators deferasirox and deferiprone for transfusional iron overload in thalassemia major: new data, new questions. Blood 2006;107:3436-41. 